\title{
Oscillatory Mixed Convention in a Porous Medium
}

\author{
M. Jana ${ }^{1}$, S. L. Maji ${ }^{1}$, S. Das ${ }^{2}$, R. N. Jana ${ }^{1}$ and S. K. Ghosh ${ }^{3 \dagger}$ \\ ${ }^{1}$ Department of Applied Mathematics, Vidyasagar University, Midnapore 721 102, West Bengal, India \\ ${ }^{2}$ Department of Mathematics, University of Gour Bnaga, Malda 732 103, West Bengal, India \\ ${ }^{3}$ Department of Mathematics, Narajole Raj College, Narajole, Midnapore 721 211, West Bengal, India \\ †Corresponding Author Email: g.swapan2002@yahoo.com
}

(Received April 26, 2012; accepted February 1, 2013)

\begin{abstract}
Oscillating mixed convection on the fully developed flow between two infinitely long vertical walls heated asymmetrically in a porous medium, has been studied. It experiences $g$-jitter force associated with microgravity in the field of space science with reference to crystal growth in space. Our present study can be exposed to a real life situation of a microgravity field. The time varying gravity field generates oscillatory free convection velocity field. This is combined with the forced oscillating flow driven by a pressure gradient.
\end{abstract}

Keywords: $g$-jitter forces, Porous medium, Darcy number, Critical Grashof number, Free convection and forced convection.

\section{NOMENClATURE}

\begin{tabular}{|c|c|c|c|}
\hline$a$ & defined in equation (15) & $P$ & dimensionless pressure \\
\hline$D_{a}$ & $\left(=k^{\prime} / L^{2}\right)$ Darcy number & $r_{T}$ & {$\left[=\left(T_{1}-T_{0}\right) /\left(T_{2}-T_{0}\right)\right]$ temperature } \\
\hline$F$ & $\begin{array}{l}\left(=u_{1} L / v\right) \text { Dimensionless velocity } \\
\text { component }\end{array}$ & $t$ & $\begin{array}{l}\text { ratio parameter } \\
\text { time }\end{array}$ \\
\hline $\operatorname{Real}\left(F e^{i \omega \tau}\right)$ & real part of $F e^{i \omega \tau}$ & $T_{0}$ & temperature at the entrance region \\
\hline$g(t)$ & $g$-jitter or time varying gravity field & $T$ & fluid temperature \\
\hline$g_{0}$ & magnitude of $g$-jitter & $T_{1}, T_{2}$ & plate temperature at the cold wall \\
\hline$G r$ & {$\left[=g_{0} \beta L^{3}\left(T_{2}-T_{0}\right) / v^{2}\right]$ Grashof number } & $u$ & $\begin{array}{c}y=0 \text { and the hot wall } y=L \\
\text { velocity component in } x \text { direction }\end{array}$ \\
\hline$(G r)_{0}$ & critical value of Grashof number & $u_{0}$ & velocity scale \\
\hline$i=\sqrt{-1}$ & complex quantity & $u_{1}$ & dimensionless velocity \\
\hline$k^{\prime}$ & permeability of a porous medium & $(x, y, z)$ & Cartesian co-ordinates \\
\hline$L$ & width of the channel & $\alpha$ & {$\left[=L^{3}\{-d P / d x\} /\left(\rho v^{2}\right)\right]$ dimensionless } \\
\hline & & & pressure gradient \\
\hline$M_{a}$ & $(=\bar{\mu} / \mu)$ viscosity ratio & $\beta$ & Coefficient of thermal expansion \\
\hline$n$ & Frequency parameter & $\eta$ & $\begin{array}{l}(=y / L) \text { non-dimensionalized width of } \\
\text { the channel }\end{array}$ \\
\hline$p$ & pressure gradient in $x$-direction & $\theta$ & $\left(=\left(T-T_{0}\right) /\left(T_{2}-T_{0}\right)\right)$ dimensionless \\
\hline$\lambda$ & defined in equation (31) & $\rho$ & $\begin{array}{l}\text { temperature } \\
\text { fluid density }\end{array}$ \\
\hline
\end{tabular}




$\begin{array}{ll}\mu & \text { viscosity of the fluid } \\ \bar{\mu} & \text { effective viscosity of the fluid saturated by } \\ & \text { porous medium } \\ v & \text { kinematic viscosity } \\ \text { 1. } & \text { INTRODUCTION }\end{array}$

An excellence of a study of a porous medium flow is the subject motivated by several important applications in science and engineering. Due to its broad range of applications in science and industry, this field has gained extensive attention lately. In a broad sense, the study of porous medium embraces fluid and thermal sciences, geothermal, petroleum and combustion engineering. Over the past decades, several studies have been taken into account of a porous medium flow with reference to Nield and Bejan (2006), Bejan (1974), Kaviany (1995), Vafai and Kim (1995) and Ingham and Pop (2002). All these authors have been studied in a prospective way to determine its flow behavior of several aspects of fluid engineering,thermal science and combustion engineering. However several studies have been taken into account of a porous medium flow. A literature survey reveals to study of Debruge and Han (1972), Du and Bilgen(1992), Hossain and Wilson (2002), Guria et al. (2009), Beg et al. (2011), Chamkha (1997), Satapathy et al. (1998), Chamkha et al. (2004), Raptis and Perdikis (2004) and Jana et al.(2012). Our present study deals with mixed convection with asymmetric heating of the wall embedded in a porous medium with reference to a study of Aung and Worku (1986). Aung and Worku (1986) studied mixed convection flow in the absence of Darcy number. The study of mixed convection flow in the presence of a magnetic field has been developed by Ghosh and Nandi (2000), Ghosh et al.(2002) and Guria et al.(2007). A recent study has been developed with $g$-jitter force to exert its influence of mixed convection flow with asymmetric heating of the wall in the presence of a magnetic field. This has been studied by Bo Pan and Ben Q (1998). However, a transverse magnetic field effect on $g$ - jitter driven flow associated with oscillating pressure gradient in a channel has been studied by Lehoczky et al. (1994), Antar and Nuotio Antar (1993) and Nelson (1991). Resently, a transient approach to radiative heat transfer free covection flow with ramped wall temperature has been analyzed by Patra et al. (2012).

The aim of the present investigation is to deal with the study of an oscillating mixed convection driven by $g$ jitter fores associated with microgravity field with a decisive importance to a highly permeable medium. A $g$ - jitter driven flow in a cavity bears direct relevance to crystal growth in space so that the net mass flow rate of the system is zero. The better model can be considered by representing the flow system of a long parallel plate channel with significant effect of pressure gradient. The practical application of unsteadiness and $\mathrm{g}$ - jitter force is of great importance to a microgravity field in taking into account of space fluid system design. In a realistic situation, the co- relation of unsteadiness and $g$ - jitter force becomes relevent to time varying gravity field driven by a time harmonic $g$ - jitter components with a frequency of oscillation and the flow at the entrance

$$
\begin{array}{ll}
\tau_{1}, \tau_{2} & \text { shear stresses at the cold wall } \eta=0 \\
& \text { and the hot wall } \eta=1 \\
\omega & \left(=n L^{2} / v\right) \text { dimensionless frequency of } \\
& \text { oscillation }
\end{array}
$$

also oscillates because of an applied pressure gradient. The representation of $g$-jitter forces is the time

varying gravity field to generate oscillatory free convection velocity field. This is combined with the forced oscillating flow driven by a pressure gradient. The importance of a study of this problem has many applications of geothermal, combustion engineering and astrospace science. We have considered $g$-jitter forces driven by oscillating mixed convection of a viscous incompressible fluid flow in a porous medium between two infinitely long vertical walls. Here we considered the Brinkman equation subject to the limit $k^{\prime} \rightarrow 0$ that gives Darcy flow and the limit $k^{\prime} \rightarrow \infty$ gives the viscouss flow, $k^{\prime}$ being the permeability of the porous medium. It is found that the velocity decreases near the channel walls while it increases at the middle of the channel with an increase in Darcy number $D_{a}$. The critical value of the buoyancy force $G r$ for which there is no flow reversal near the cold wall and it decreases with an increase in Darcy number $D_{a}$.

\section{MATHEMATICAL FORMULATION AND ITS SOLUTION}

Consider the mixed convection of viscous incompressible fluid flow in a porous medium between two infinitely long vertical walls separated by a distance $L$. The fluid is at temperature $T_{0}$ and flows due to temperature and pressure gradient in between two parallel walls. The walls are infinitely long along $x$-axis and $z$-direction with different wall temperatures [see Fig.1]. We consider the fully developed unsteady flow in a porous medium. We assume that the g-jitter field under consideration is spatially constant and otherwise varies with time harmonically.

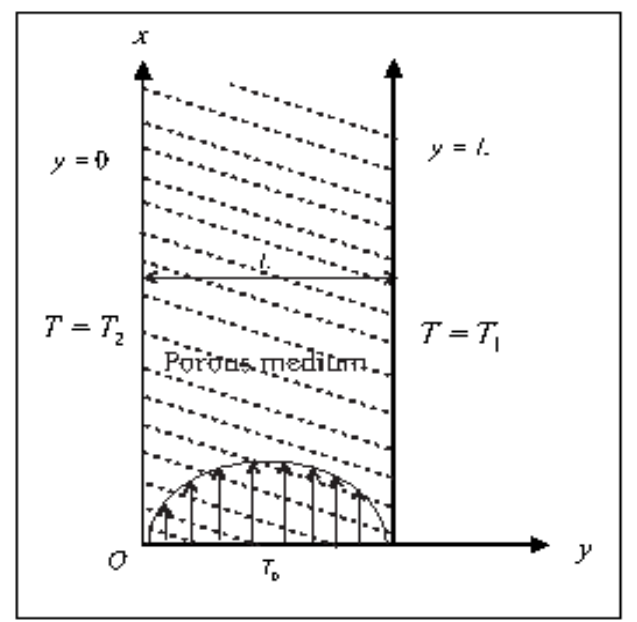

Fig. 1. Geometry of the problem 
Further, the flow at the entrance of the channel also oscillates harmonically due to an applied pressure gradient. An oscillatory free convection velocity will generate because of the variation of the gravity field. Thus, the problem becomes a free and forced convection oscillating flow in the presence of pressure gradient.

The equation of motion under the usual Boussinesq approximation is

$$
\frac{\partial u}{\partial t}=-\frac{1}{\rho} \frac{\partial p}{\partial x}+\frac{\bar{\mu}}{\rho} \frac{d^{2} u}{d y^{2}}+g(t) \beta\left(T-T_{\infty}\right)-\frac{\mu}{\rho k^{\prime}} u,
$$

where $k^{\prime}$ the permeability of the porous medium, $\beta$ the thermal expansion co-efficient, $\mu$ the fluid viscosity, $\bar{\mu}$ the effective viscosity of the fluid saturated by porous medium and $\rho$ the fluid density.

The energy equation is

$$
\frac{d^{2} T}{d y^{2}}=0
$$

The velocity and temperature boundary conditions are

$u=0 \quad$ at $\quad y=0$ and $u=0$ at $y=L$,

$T=T_{1} \quad$ at $\quad y=0$ and $T=T_{2} \quad$ at $\quad y=L$.

Assuming

$u=u_{1}(y) e^{i n t}, \quad g(t)=g_{0} e^{i n t}, \quad p=P(x) e^{i n t}$,

the Eq. (1) becomes

$$
\text { in } u_{1}=-\frac{1}{\rho} \frac{d P}{d x}+\frac{\bar{\mu}}{\rho} \frac{d^{2} u_{1}}{d y^{2}}+g_{0} \beta\left(T-T_{0}\right)-\frac{\mu}{\rho k^{\prime}} u_{1} .
$$

Introducing non-dimensional variables

$\eta=\frac{y}{L}, F=\frac{u_{1} L}{v}, \theta=\frac{T-T_{0}}{T_{2}-T_{0}}$

Eqs. (6) and Eq. (2) become

$$
\begin{aligned}
& \frac{d^{2} F}{d \eta^{2}}-\left(\frac{1}{M a D a}+i \omega\right) F=-(\alpha+G r \theta), \\
& \frac{d^{2} \theta}{d \eta^{2}}=0,
\end{aligned}
$$

where $G r=\frac{g_{0} \beta L^{3}\left(T_{2}-T_{0}\right)}{v^{2}}$ is the Grashof number, $\omega=\frac{n L^{2}}{v}$ the frequency parameter, $\alpha=\frac{L^{3}}{\rho v^{2}}\left(-\frac{d P}{d x}\right)$ the non-dimentional pressure gradient, $M a=\frac{\bar{\mu}}{\mu} \quad$ the viscosity ratio and $D_{a}=\frac{k^{\prime}}{L^{2}}$ the Darcy number. It may be noted that the limit $D_{a} \rightarrow \infty$ gives a clear fluid and the limit $D_{a} \rightarrow 0$ gives the unmitigated Darcy flow.

The corresponding boundary conditions for $F(\eta)$ and $\theta(\eta)$ are
$F(0)=0=F(1)$

$\theta(0)=r_{T}, \theta(1)=1$,

where $r_{T}=\frac{T_{1}-T_{0}}{T_{2}-T_{0}}$ is temperature ratio parameter.

The solution of the Eq. (9) subject to the boundary conditions Eq. (11) is

$\theta(\eta)=\left(1-r_{T}\right) \eta+r_{T}$.

On the use of Eq. (12), the Eq. (8) becomes

$$
\frac{d^{2} F}{d \eta^{2}}-\left(\frac{1}{M_{a} D_{a}}+i \omega\right) F=-\left[\left(\alpha+G r r_{T}\right)+G r\left(1-r_{T}\right) \eta\right] .
$$

The solution of the Eq. (13) subject to the boundary conditions Eq. (10) is

$$
\begin{aligned}
& F(\eta)=\frac{1}{a^{2}}\left[\left(\alpha+G r r_{T}\right)\left\{1-\frac{\sinh a \eta}{\sinh a}-\frac{\sinh a(1-\eta)}{\sinh a}\right\}\right. \\
& \left.+\operatorname{Gr}\left(1-r_{T}\right)\left\{\eta-\frac{\sinh a \eta}{\sinh a}\right\}\right],
\end{aligned}
$$

where

$a=\left(\frac{1}{M_{a} D_{a}}+i \omega\right)^{\frac{1}{2}}=\alpha_{1}+i \beta_{1}$,

$\alpha_{1}, \beta_{1}=\frac{1}{\sqrt{2}}\left[\left\{\frac{1}{\left(M_{a} D_{a}\right)^{2}}+\omega^{2}\right\}^{\frac{1}{2}} \pm \frac{1}{M_{a} D_{a}}\right]^{\frac{1}{2}}$.

The pressure gradient $\alpha$ is determined by requiring that the flow satisfies the following condition, $\int_{0}^{1} F(\eta) d \eta=1$.

On the use of the Eq. (14) in the above equation, the mass conservation equation for $\alpha$ as

$\alpha=\frac{a^{3} \sinh a}{a \sinh a+2(1-\cosh a)}-\frac{1}{2} G r\left(1+r_{T}\right)$.

If the rate of mass flow is zero, the pressure pressure gradient $\alpha$ reduces to

$\alpha=-\frac{1}{2} G r\left(1+r_{T}\right)$.

On the use of Eq. (16), the Eq. (14) becomes

$$
\begin{aligned}
& F(\eta)=\frac{1}{a^{2}}\left[\left\{\frac{a^{3} \sinh a}{a \sinh a+2(1-\cosh a)}-\frac{1}{2} \operatorname{Gr}\left(1-r_{T}\right)\right\}\right. \\
& \left.\times\left\{1-\frac{\sinh a \eta}{\sinh a}-\frac{\sinh a(1-\eta)}{\sinh a}\right\}+G r\left(1-r_{T}\right)\left\{\eta-\frac{\sinh a \eta}{\sinh a}\right\}\right]
\end{aligned}
$$

It is interesting to note that the velocity distribution given by Eq. (18) for $G r=0$ (the pure forced 
convection) at any values of $r_{T}$ and for $r_{T}=1$ for any values of $G r$ are identical and the solution for $F(\eta)$ given by Eq. (18), reduces to

$$
\begin{aligned}
& F(\eta)=\frac{1}{a^{2}}\left[\frac{a^{3} \sinh a}{a \sinh a+2(1-\cosh a)}\right. \\
& \left.\times\left\{1-\frac{\sinh a \eta}{\sinh a}-\frac{\sinh a(1-\eta)}{\sinh a}\right\}\right]
\end{aligned}
$$

In a free convection process, substituting $\alpha=0$ into the solution Eq. (14), we have

$$
\begin{aligned}
& F(\eta)=\frac{G r}{a^{2}}\left[r_{T}\left\{1-\frac{\sinh a \eta}{\sinh a}-\frac{\sinh a(1-\eta)}{\sinh a}\right\}\right. \\
& \left.+\left(1-r_{T}\right)\left\{\eta-\frac{\sinh a \eta}{\sinh a}\right\}\right] .
\end{aligned}
$$

In the limit $D_{a} \rightarrow \infty$ (for a clear fluid), $\omega \rightarrow 0$ and $r_{T}=1$ the Eq. (20) becomes

$$
F(\eta)=\frac{1}{2} G r\left(\eta-\eta^{2}\right) .
$$

In the vicinity of the central section of the channel, the velocity is given by Eq. (21) reduces to

$$
F(\eta)=\frac{1}{8} G r .
$$

We shall now discuss some limiting cases:

\subsection{Soluton $D_{a}>>1$ and $\omega \ll 1$}

The velocity distribution and the pressure gradient are given by Eq. (14) and Eq. (16) respectively, become

$$
\begin{aligned}
& F(\eta)=6\left(\eta-\eta^{2}\right)-\frac{1}{12} G r\left(1-r_{T}\right)\left(\eta-3 \eta^{2}+2 \eta^{3}\right) \\
& +\left(\frac{1}{D_{a}}+i \omega\right)\left[\frac{1}{10}\left(\eta-6 \eta^{2}+10 \eta^{3}-5 \eta^{4}\right)\right. \\
& \left.+\frac{1}{720} G r\left(1-r_{T}\right)\left(\eta-10 \eta^{3}+15 \eta^{4}-6 \eta^{5}\right)\right]
\end{aligned}
$$

and

$$
\alpha=12\left\{1+\frac{1}{10}\left(\frac{1}{D_{a}}+i \omega\right)\right\}-\frac{1}{2} G r\left(1+r_{T}\right) .
$$

In the limit $D_{a} \rightarrow \infty$, the Eq. (23) becomes

$$
\begin{aligned}
& F(\eta)=6\left(\eta-\eta^{2}\right)-\frac{1}{12} G r\left(1-r_{T}\right)\left(\eta-3 \eta^{2}+2 \eta^{3}\right) \\
& +\frac{i \omega}{720}\left[72\left(\eta-6 \eta^{2}+10 \eta^{3}-5 \eta^{4}\right)\right. \\
& \left.+G r\left(1-r_{T}\right)\left(\eta-10 \eta^{3}+15 \eta^{4}-6 \eta^{5}\right)\right]
\end{aligned}
$$

The velocity distribution given by Eq. (23) is dependent on Grashof number, Darcy number and frequency of oscillation. For $\mathrm{r}_{\mathrm{T}}=1$ ( uniform temperature variation along the wall) the velocity distribution is closely resemblance to a forced convection flow due to absence of Grashof number. Hence the velocity distribution is independent of Grashof number but dependent on Darcy number and frequency of oscillation. The expression for pressure gradient given by Eq. (24) plays a significant role with reference to temperature ratio parameter $r_{T}$ so that the effect of buoyancy force becomes predominant for the constant rate of flow. The expression for the velocity distribution given by Eq. (25) leads to the clear fluid and the buoyancy force accelerates the entire flow situation on the velocity field.

\subsection{Soluton for an oscillating mixed convection with Darcy number $D_{a} \rightarrow \infty$ (clear fluid)}

The Eq. (14) together with the expression Eq.(16) yields

$$
\begin{aligned}
& F(\eta)=\frac{1}{i \omega}\left[\frac{(i \omega)^{\frac{3}{2}} \sinh \sqrt{i \omega}}{\sqrt{i \omega} \sinh \sqrt{i \omega}+2(1-\cosh \sqrt{i \omega})}\right. \\
& \times\left\{1-\frac{\sinh \sqrt{i \omega} \eta}{\sinh \sqrt{i \omega}}-\frac{\sinh \sqrt{i \omega}(1-\eta)}{\sinh \sqrt{i \omega}}\right\} \\
& -\frac{1}{2} G_{r}\left(1-r_{T}\right)\left\{1-2 \eta+\frac{\sinh \sqrt{i \omega} \eta}{\sinh \sqrt{i \omega}}-\frac{\sinh \sqrt{i \omega}(1-\eta)}{\sinh \sqrt{i \omega}}\right\} .
\end{aligned}
$$

If frequency of the oscillations is very large, the above Eq. (26) becomes

$$
\begin{aligned}
& F(\eta)=\frac{\sqrt{i \omega}}{\sqrt{i \omega}-2}\left[1-e^{-\sqrt{i \omega}(1-\eta)}-e^{-\sqrt{i \omega} \eta}\right] \\
& -\frac{1}{2 i \omega} G r\left(1-r_{T}\right)\left[1-2 \eta+e^{-\sqrt{i \omega}(1-\eta)}-e^{-\sqrt{i \omega} \eta}\right] .
\end{aligned}
$$

At the limit of $\omega \rightarrow \infty$, we have

$\lim _{\omega \rightarrow \infty} F(\eta)=1-e^{-\sqrt{i \omega} \eta}-e^{-\sqrt{i \omega}(1-\eta)}$.

If the frequency of oscillations is very large the expression for the velocity given by Eq. (27) is an exponential in nature with the frequency of oscillations and Grashof number plays an important role in determining the flow behavior. In this situation, the frequency of oscillations survives exponentially on the velocity field with significant effect of buoyancy force. The Eq. (28) shows that a thin stokes layer form near the walls for large frequency of the oscillating flows. The thickness of the layer is $\mathrm{O}\left\{(2 / \omega)^{\frac{1}{2}}\right\}$ 
corresponding to decrease with an increase in frequency of oscillations.

\section{RESUltS AND DISCUSSION}

The quantity plotted along the vertical axis is $\operatorname{Imag}\left(F e^{i \omega \tau}\right)=\operatorname{Real}(F) \cos \omega \tau+\operatorname{Imag}(F) \sin \omega \tau$,

which represents the measurable value for a driving force $g=\operatorname{Im} a g\left(g_{0} e^{i \omega \tau}\right)=g_{0} \sin \omega \tau$ and $u_{o}(\tau)=u_{0} \sin \omega \tau$, where Imag stands for imaginary part. It is noted that form Fig. 2 that at around $\omega \tau=\pi$ and $\omega \tau=2 \pi$ the velocity profile oscilattes along the width of the channel but for other times the velocity profile is approximately parabolic, as expcted for the symetrically heated walls $\left(r_{T}=1\right)$ and for forecd convection in a channel. It is evident fron Fig. 3 that the profiles are skewed near the cold wall as expected for higher buoyancy while the velocity profiles are of oscillatory character near the cold wall due to asymmetric heating of the wall whereas the velocity profiles oscillate at around $\omega \tau=\pi$ and $\omega \tau=2 \pi$ along the width of the channel.

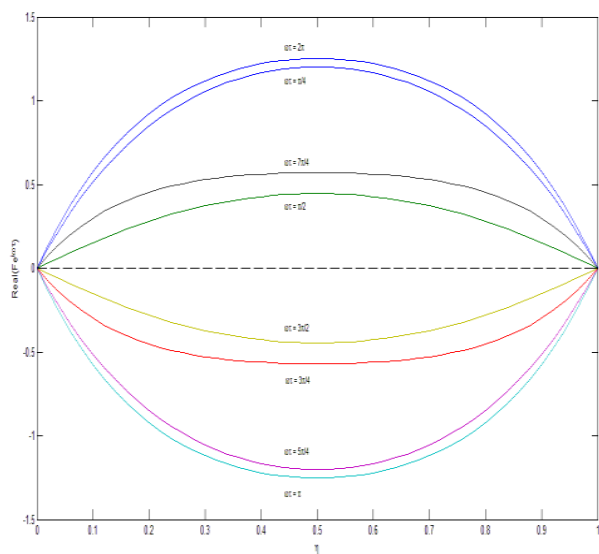

Fig. 2. Velocity profile for $\omega \tau$ with $r_{T}=1$, $G r=20, D_{a}=0.05, \omega=10, M_{a}=1$

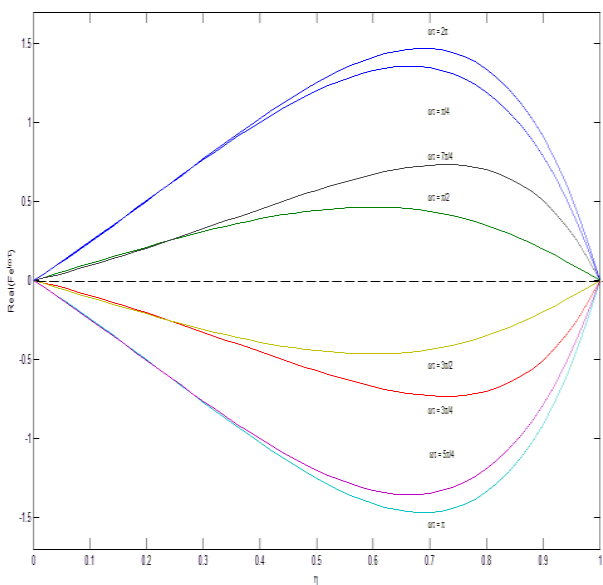

Fig. 3. Velocity profile for $\omega \tau$ with $r_{T}=0.2$,

$$
G r=100, \quad D_{a}=0.05, \omega=10, M_{a}=1
$$

Fig. 4 demonastrates that at higher buoyancy $(G r=100)$ the profile becomes parabolic at $r_{T}=1$. This indicates that the flow is characterised by a unifrom temperature distribution (symmetrically heated wall) when $r_{T}=1$. In the case of asymmetric heating of the wall the velocities increase at the cold wall while the velocities decrease at the hot wall with increase in $r_{T}$ whereas at each value of $r_{T}$, every profile coincides with the mid plane of the central section of the channel.

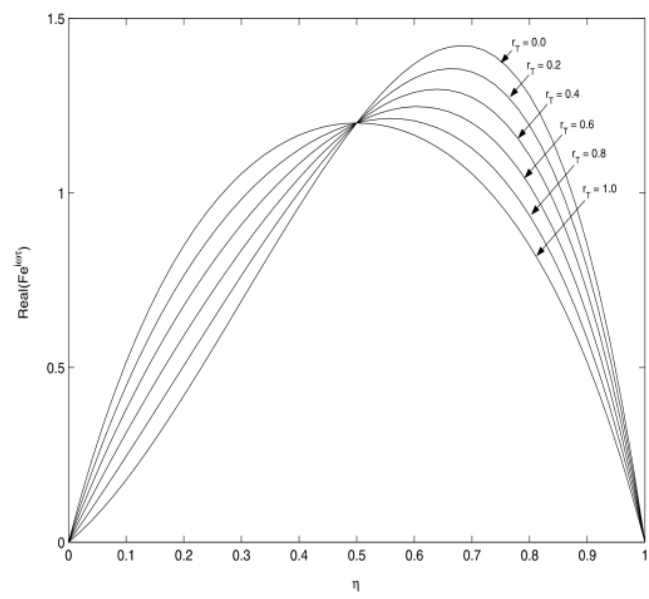

Fig. 4. Velocity profile for $r_{T}$ with $D_{a}=0.05$,

$$
G r=100, \omega=10, \omega \tau=\frac{\pi}{4}, M_{a}=1
$$

It is noticed from Fig. 5 that the effect of Darcy number leads to increase the velocities near the cold wall as well as near the hot wall while the effect of Darcy number becomes predominant over the central region. In the central region, the velocities are markedly increased with increase in Darcy number. This situation reveals that the velocities depend on permeability of the porous medium to boost up the velocities in the central region for moderate values of Darcy number. Fig. 6 reveals that the velocity profile becomes parabolic when the buoyancy force is absent $(G r=0)$.

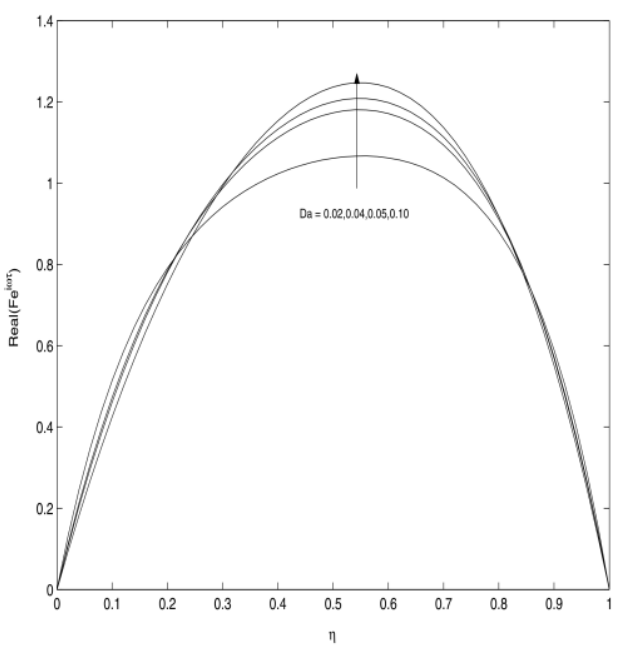

Fig.5. Velocity profile for $D_{a}$ with $r_{T}=0.2$,

$$
G r=20, \omega=10, \omega \tau=\frac{\pi}{4}, M_{a}=1
$$

It is seen from Fig. 6 that the velocity increases near the hot wall with increase in Grashof number $G r$ while a flow reversal occurs near the cold wall with increase in 
Grashof number $G r$ with reference to higher buonyancy $G r \geq 300$. The nature of velocity profile on Fig. 6 is closely resemblance to the study of Aung and Worku (1986).

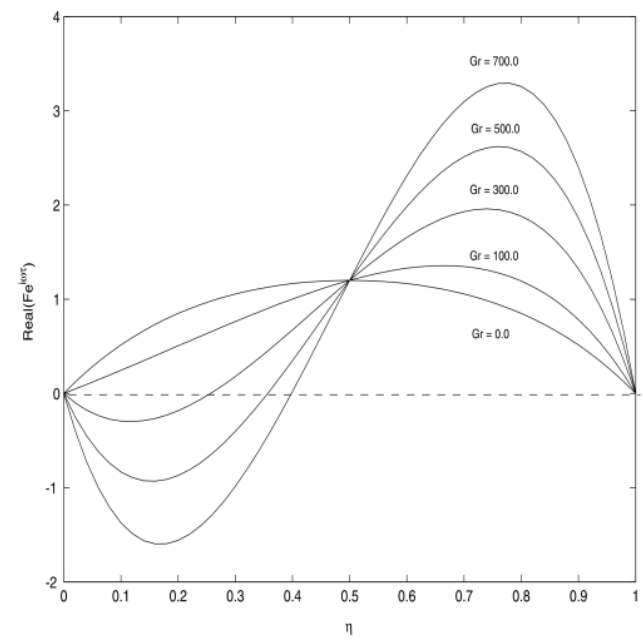

Fig.6. Velocity profile for $G r$ with $r_{T}=0.2, \quad \omega=10$,

$$
D_{a}=0.05, \omega \tau=\frac{\pi}{4}, M_{a}=1
$$

The shear stresses at the cold wall $\eta=0$ and the hot wall $\eta=1$ are respectively given by $\tau_{1}=\operatorname{Real}\left\{\left(\frac{d F}{d \eta}\right)_{\eta=0} e^{i \omega \tau}\right\}$ and $\tau_{2}=\operatorname{Real}\left\{\left(\frac{d F}{d \eta}\right)_{\eta=1} e^{i \omega \tau}\right\}$,

where

$$
\begin{aligned}
& \left(\frac{d F}{d \eta}\right)_{\eta=0}=-\frac{1}{a^{2} \sinh a}[\lambda a(1-\cosh a) \\
& \left.+\frac{1}{2} \operatorname{Gr}\left(1-r_{T}\right)\{a(1+\cosh a)-2 \sinh a\}\right],
\end{aligned}
$$$$
\left(\frac{d F}{d \eta}\right)_{\eta=1}=\frac{1}{a^{2} \sinh a}[\lambda a(1-\cosh a)
$$$$
\left.+\frac{1}{2} G r\left(1-r_{T}\right)\{2 \sinh a-a(1+\cosh a)\}\right]
$$

where

$$
\lambda=\frac{a^{3} \sinh a}{a \sinh a+2(1-\cosh a)}
$$

and $a$ is given by Eq. (15).

The numerical values of the shear stresses $\tau_{1}$ and $\tau_{2}$ at the wall $\eta=0$ and $\eta=1$ are shown in the Figs.7-8 against $r_{T}$ for several values of $\omega$ and $D_{a}$ when $M a=1$. Figure 7 shows that the shear stress $\tau_{1}$ at the wall $\eta=0$ and the magnitude of the shear stress $\tau_{2}$ at the wall $\eta=1$ increases with an increase in frequency parameter $\omega$. It is remarked here that the permeability of the porous medium plays an important roll in determining the nature of shear stress when the frequency of oscillation $\omega$ is taken into account. In turn the effects of shear stresses become relevant to the case of an inverse permeability of the porous medium propelled by the viscosity of the fluid so that shear stresses increase with increase in frequency parameter $\omega$. It is noticed form Fig. 8 that the shear stress $\tau_{1}$ at the wall $\eta=0$ and the magnitude of the shear stress $\tau_{2}$ at the wall $\eta=1$ decreases with an increase in Darcy number $D_{a}$. Thus it arrives at an interesting conclusion that the permeability of the porous medium leads to slow down the particle so that shear stresses decrease with increase on Darcy number. Further, for fixed value of $\omega$ and $D a, \tau_{1}$ steadily increases while magnitude of $\tau_{2}$ steadily decreases with an increase in temperature ratio parameter $r_{T}$.

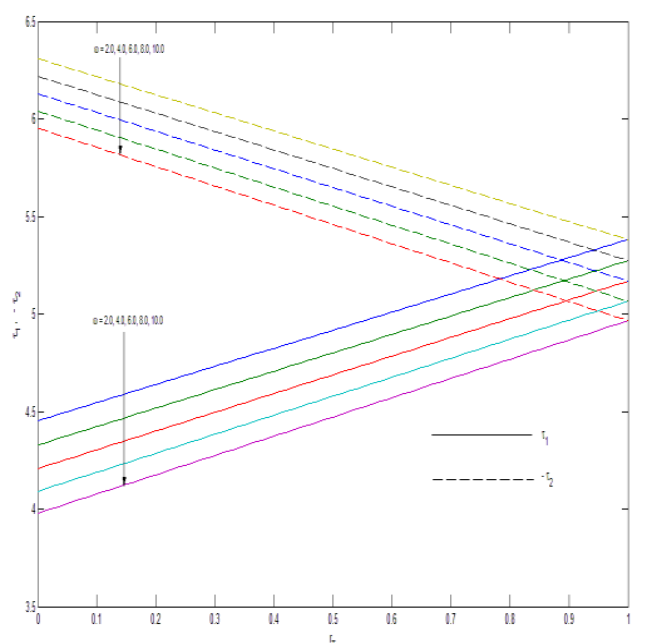

Fig.7. Shear stresses $\tau_{1}$ and $\tau_{2}$ at the walls $\eta=0, \eta=1$ for $\omega$ with $D_{a}=0.05, G r=20, \omega \tau=\frac{\pi}{4}$

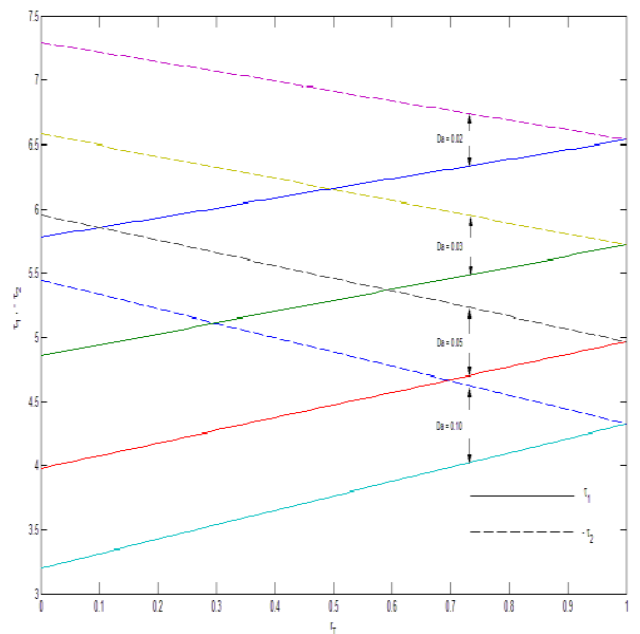

Fig. 8. Shear stresses $\tau_{1}$ and $\tau_{2}$ at the walls $\eta=0, \eta=1$ for $D_{a}$ with $G r=20, \omega=10, \omega \tau=\frac{\pi}{4}$ 
The critical value of $G r_{0}$ for which there is no flow reversal near the cold wall $\eta=0$ to obtained from $\left(\frac{d F}{d \eta}\right)_{\eta=0}=0$, which is

$$
G r_{0}=-\operatorname{Real}\left[\frac{2 \lambda a(1-\cosh a)}{\left(1-r_{T}\right)[a(1+\cosh a)-2 \sinh a]}\right],
$$

where $a$ is given by Eq. (15) and the corresponding value of the critical Grashof number at the hot wall $\eta=1$ is given by $G r_{1}=-G r_{0}$. The values of the critical Grashof number $G r_{0}$ are entered in Table 1 for different values of $D_{a}$ and $r_{T}$. It is seen from Table 1 that the critical Grashof number $G r_{0}$ at the wall $\eta=0$ increases with increase in the temperature ratio parameter $r_{T}$ whereas it decreases with increase in the Darcy number $D_{a}$. Although buoyancy force accelerates the fluid particle to become flow reversal at higher buoyancy [see Fig. 6]. Critical Grashof number is obtained where no flow reversal occurs at the cold wall but a tendency of increasing remains unaltered.

Table 1 Variation of $10^{-3} \mathrm{Gr}_{0}$

\begin{tabular}{|c|c|c|c|c|}
\hline$r_{T} \backslash D a$ & 0.02 & 0.04 & 0.05 & 0.01 \\
\hline 0.0 & 0.1935 & 0.1328 & 0.1206 & 0.0961 \\
0.2 & 0.2419 & 0.1660 & 0.1507 & 0.1201 \\
0.4 & 0.3226 & 0.2214 & 0.2010 & 0.1602 \\
0.6 & 0.4839 & 0.3321 & 0.3015 & 0.2403 \\
\hline
\end{tabular}

The values of the pressure gradient $\alpha$ are entered in Table 2 for different values of $D_{a}$ and $r_{T}$. It is seen from Table 2 that the pressure gradient $\alpha$ decreases with increase in both the temperature ratio parameter $r_{T}$ and Darcy number $D_{a}$.

Table 2 Variation of $10^{-3} \alpha$

\begin{tabular}{|c|c|c|c|c|}
\hline$r_{T} \backslash D a$ & 0.02 & 0.04 & 0.05 & 0.01 \\
\hline 0.0 & 0.0597 & 0.0314 & 0.0256 & 0.0139 \\
0.2 & 0.0577 & 0.0294 & 0.0236 & 0.0119 \\
0.4 & 0.0557 & 0.0274 & 0.0216 & 0.0099 \\
0.6 & 0.0537 & 0.0254 & 0.0196 & 0.0079 \\
\hline
\end{tabular}

\section{CONCLUSION}

Oscillatory mixed convection viscous incompressible flow in porous medium between two infinitely long vertical walls is considered. It is found that the velocity profile is almost parabolic in nature for the small value of buoyancy force $G r$ while the skewness is characterised by the higher buoyancy and at large value of $\mathrm{Gr}$ there arise flow reversal near the cold wall. It is found that the velocity profile increases near the cold wall of the channel while it decreases near the hot wall of that channel with increase in temperature ratio parameter $r_{T}$. In the presence of Darcy number, the magnitude of the shear stresses $\tau_{1}$ at the wall $\eta=0$ increases while that of the shear stress $\tau_{2}$ at the wall $\eta=1$ decreases with increase in $r_{T}$. The critical Grashof number, for which there is no flow reversal near the wall $\eta=0$ and it decreases with increase in Darcy number.

\section{REFERENCES}

Aung, W. and G. Worku (1986). Theory of fully developed, combined convection including flow reversal. Journal of Heat Transfer, 108, 485-488.

Beg, O.A., S.K. Ghosh and M. Narahari (2011). Mathematical modeling of oscillatory MHD Couette flow in a rotating highly permeable medium permeated by an oblique magnetic field. Chemical Engineering and Communications, 198, 235-254.

Bejan, A.(1994). Convection heat transfer, Weiley, New York.

Chamka, A.J. (1997). Solar radiation assisted natural convection in a uniform porous medium supported by a vertical flat plate. ASME J. Heat Transfer, 119, 89-96.

Chamka, A.J., H.S.Takhar and O.A. Beg (2004). Radiative free convective non-Newtonian fluid flow past a wedge embedded in a porous medium. Int. J. Fluid Mechanics Research, 31,101-115.

Debruge, L.L. and L.S. Han (1972). Heat tranfer in a channel with a porous wall for turbine cooling application. J. Heat Transfer, 94, 385-390.

Du, Z.-G. and E.Bilgen (1992). Natural convection in vertical cavities with internal heat generating porous medium. Wärme-und Stoffübertr, 27, 149155.

Ghosh, S.K. and D.K. Nandi (2000). Magnetohydrodynamic fully developed combined convection flow between vertical plates heated asymmetrically. J. Technical Physics, 41, 173-185.

Ghosh, S.K., I. Pop and D.K. Nandi (2002). MHD fully developed mixed convection flow with asymmetric heating of the wall. Int. J. Applied Mechanics and Engineering, 7, 1211-1228.

Guria, M., B.K. Das, R.N. Jana and S.K. Ghosh (2007). Effects of wall conductance on MHD fully developed flow with asymmetric heating of the wall. Int. J. Fluid Mechanics Research, 34, 521534.

Guria, M., R.N. Jana, S.K. Ghosh and I. Pop (2009). Three dimensional free convection flow in a vertical channel filled with a porous medium. Journal of Porous Media, 12, 985-995.

Hossain, M.A. and M. Wilson (2002). Natural convection flow in a fluid-saturated porous medium enclosed by non-isothermal walls with 
M. Jana et al. / JAFM, Vol. 7, No. 1, pp. 43-50, 2014.

heat generation. Int. J. Thermal Science, 41, 447454 .

Ingham, D.B. and I. Pop (2002). Transportphenomena in porous media-II, Elsevier science Ltd., The Boulevard, Langford Lane, Kindlington, Oxford OX5, 1GB, UK.

Jana, M., S. Das, S.L. Maji, R.N. Jana and S.K. Ghosh (2012). Natural convection boundary layer flow past a flat plate of finite dimensions. Journal of Porous Media, 15(6), 585 - 593.

Kaviany, M.(1995). Principles of heat transfer in porous media, Springer-Verlay, New York, Inc.

Neild, D.A. and A. Bejan (2006). Convection in Porous Media. Third Edition. New York, Springer Science+Business Media, Inc., 2006.

Pan, B. and B.Q. Li (1998). Effects of magnetic fields in oscillating mixed convection. Int. J. Heat and Mass Transfer, 41, 2075-2710.
Patra, R.R., S. Das, R.N. Jana and S.K. Ghosh (2012). Transient approach to radiative heat transfer free covection flow with ramped wall temperature, Journal of Applied Fluid Mechanics, 5(2), 9-13.

Raptis, A. and C. Perdikis (2004). Unsteady flow through porous medium in the presence of radiation, Transport Porous Media, J(57), 171179.

Satapathi, S., A. Bedford, S. Bless and A. Raptis (1998). Radiation and free convection flow through a porous medium, Int. Comm. Heat Mass Transfer, 25, 289-297.

Vafai, K. and S.J. Kim (1995). On the limitations of the Brinkman-Forchheimer-extended Darcy equation. Int. J. Heat Fluid Flow, 16, 11-15. 